\title{
Neoplasia de cabeça e pescoço: Principais causas e tratamentos
}

\author{
Head and neck neoplasm: Main causes and treatments \\ Neoplasia de cabeza y cuello: Principales causas y tratamientos
}

Recebido: 15/04/2021 | Revisado: 25/04/2021 | Aceito: 03/05/2021 | Publicado: 16/05/2021

Lennara Pereira Mota

ORCID: https://orcid.org/0000-0002-2629-6634

Centro Universitário Maurício de Nassau, Brasil

E-mail: lennaramota@hotmail.com

Millena Raimunda Martins de Almeida Carvalho

ORCID: https://orcid.org/0000-0003-2431-2431 Universidade Federal do Piauí, Brasil

E-mail: millenamartinsalmeida2@gmail.com

Amadeu Luis de Carvalho Neto

ORCID: https://orcid.org/0000-0001-7726-6120

Universidade Federal do Piauí, Brasil

E-mail: amadeusc.neto@ hotmail.com

Francis Aiala De Araújo Ferreira

ORCID: https://orcid.org/0000-0001-7301-0638

Universidade Ceuma, Brasil

E-mail: francisaiala@hotmail.com

Josefa Angélica Cerqueira Poty

ORCID: https://orcid.org/0000-0002-3838-0833

Centro Universitário Unifacid, Brasil

E-mail: potyjosefa@gmail.com

José Guilherme Férrer Pompeu

ORCID: https://orcid.org/0000-0001-9038-0833

Universidade Federal do Piauí, Brasil

E-mail: gpompeu8@gmail.com

Marcela Maria Lopes Rocha

ORCID: https://orcid.org/0000-0002-0189-3838

Instituto de Ensino Superior do Vale do Parnaíba, Brasil

E-mail: mamalrocha@outlook.com

Rodrigo Campelo de Moura Fé

ORCID: https://orcid.org/0000-0003-4776-078X

Instituto de Ensino Superior do Vale do Parnaíba, Brasil

E-mail: rodrigocmourafe@hotmail.com

Luiz Eduardo Batista da Silva

ORCID: https://orcid.org/0000-0002-7225-1664

Instituto de Ensino Superior do Vale do Parnaíba, Brasil

E-mail: Luizleduardo@ hotmail.com

Edmilson Alves de Carvalho Neto

ORCID: https://orcid.org/0000-0001-5702-2954

Instituto de Ensino Superior do Vale do Parnaíba, Brasil

E-mail: edmilsonacn@ hotmail.com

Francisca Mikaelly De Sousa Silva

ORCID: https://orcid.org/0000-0001-7485-7048 Centro Universitário Maurício de Nassau, Brasil

E-mail: mikaellysousa235@gmail.com

Carla Patrícia de Carvalho Oliveira

ORCID: https://orcid.org/0000-0002-0336-3347

Departamento de Medicina Comunitária, Brasil

E-mail: Carlapatricia@Ufpi.edu.br

Mirian Nascimento Rabelo

ORCID: https://orcid.org/0000-0003-4843-2251

Universidade Federal do Piauí, Brasil

E-mail: Mirian.rabelo@hotmail.com

Lillian Lettiere Bezerra Lemos Marques

ORCID: https://orcid.org/0000-0002-8261-8589

Centro Universitário Maurício de Nassau, Brasil

E-mail: lillianlbl@gmail.com

Maria Grazielly de Sousa Oliveira

ORCID: https://orcid.org/0000-0003-1175-5997

Universidade Estadual do Piauí, Brasil

E-mail: graziellyolive@ hotmail.com 


\author{
Iracely de Vasconcelos Cruz \\ ORCID: https://orcid.org/0000-0002-8942-1324 \\ Faculdade Estácio de Teresina, Brasil \\ E-mail: Iracely.cruz@ hotmail.com
}

\begin{abstract}
Resumo
O termo câncer de cabeça e pescoço (CCP) refere-se a um grupo de tumores heterogêneos que acometem o trato gastrointestinal superior, sendo o principal tipo histológico, o carcinoma espinocelular, responsável por cerca de $90 \%$ dos casos. O trabalho tem por objetivo expor através da análise de artigos científicos as principais causas e tratamentos associados à Neoplasia de cabeça e pescoço. Trata-se de uma revisão bibliográfica de caráter qualitativo que se baseia na produção científica a partir de estudos já publicados entre os anos de 2017 a 2021. No câncer de cabeça e pescoço, fatores de risco genéticos e ambientais contribuem para sua etiologia, como beber e fumar, que são os fatores de risco mais relevantes para doenças do trato gastrointestinal superior. No que diz respeito ao cancro da tiroide, são múltiplas as razões consideradas fatores de risco, nomeadamente exposição à radiação, condições hormonais, obesidade, história familiar de cancro e ingestão de alimentos iodados. Os tratamentos para esses tipos de câncer podem incluir ressecção cirúrgica, quimioterapia, radioterapia, terapia hormonal ou uma combinação de múltiplas modalidades de tratamentos, resultando em alterações na função e na qualidade de vida. São necessários estudos científicos mais aprofundados sobre esse câncer e o desenvolvimento de medidas de prevenção para a população em geral, conscientizando-os sobre como o tabagismo, o consumo de bebidas alcoólicas e a infecção pelo o HPV podem ser causas para o desenvolvimento dessa neoplasia maligna.
\end{abstract}

Palavras-chave: Câncer; Carcinoma de cabeça e pescoço; Tumores; Tratamento.

\begin{abstract}
The term head and neck cancer (CCP) refers to a group of heterogeneous tumors that affect the upper gastrointestinal tract, the main histological type of which is squamous cell carcinoma, responsible for about $90 \%$ of cases. The work aims to expose through the analysis of scientific articles the main causes and treatments associated with neoplasia of the head and neck. This is a qualitative bibliographic review based on scientific production based on studies already published between the years 2017 to 2021 . In head and neck cancer, genetic and environmental risk factors contribute to its etiology, such as drinking and smoking, which are the most relevant risk factors for diseases of the upper gastrointestinal tract. With regard to thyroid cancer, there are multiple reasons considered to be risk factors, namely radiation exposure, hormonal conditions, obesity, family history of cancer and ingestion of iodized foods. Treatments for these types of cancer can include surgical resection, chemotherapy, radiation therapy, hormonal therapy or a combination of multiple treatment modalities, resulting in changes in function and quality of life. More in-depth scientific studies are needed on this cancer and the development of preventive measures for the general population, making them aware of how smoking, alcohol consumption and HPV infection can be causes for the development of this malignant neoplasm.
\end{abstract}

Keywords: Cancer; Head and neck carcinoma; Tumors; Treatment.

\title{
Resumen
}

El término cáncer de cabeza y cuello (CCP) se refiere a un grupo de tumores heterogéneos que afectan al tracto gastrointestinal superior, cuyo principal tipo histológico es el carcinoma de células escamosas, responsable de aproximadamente el $90 \%$ de los casos. El trabajo tiene como objetivo exponer a través del análisis de artículos científicos las principales causas y tratamientos asociados a las neoplasias de cabeza y cuello. Se trata de una revisión bibliográfica cualitativa basada en la producción científica basada en estudios ya publicados entre los años 2017 a 2021 . En el cáncer de cabeza y cuello, los factores de riesgo genéticos y ambientales contribuyen a su etiología, como beber y fumar, que son los de riesgo más relevantes. factores de enfermedades del tracto gastrointestinal superior. Con respecto al cáncer de tiroides, existen múltiples razones que se consideran factores de riesgo, a saber, la exposición a la radiación, las condiciones hormonales, la obesidad, los antecedentes familiares de cáncer y la ingestión de alimentos yodados. Los tratamientos para estos tipos de cáncer pueden incluir resección quirúrgica, quimioterapia, radioterapia, terapia hormonal o una combinación de múltiples modalidades de tratamiento, lo que resulta en cambios en la función y la calidad de vida. Se necesitan estudios científicos más profundos sobre este cáncer y el desarrollo de medidas preventivas para la población en general, concienciando cómo el tabaquismo, el consumo de alcohol y la infección por VPH pueden ser causas del desarrollo de esta neoplasia maligna.

Palabras clave: Cáncer; Carcinoma de cabeza y cuello; Tumores; Tratamiento.

\section{Introdução}

Tumor é definido como uma massa anormal de tecido causada pelo crescimento autônomo excessivo e desordenado de um grupo de células (independente dos fatores de crescimento e mecanismos reguladores que existem nos tecidos normais, a estrutura formada por este tipo de célula é diferente dos tecidos considerados normais). Quando a estimulação inicial do 
crescimento cessa, essa proliferação celular persiste. Considerando o seu comportamento clínico, esta neoplasia é caracterizada pela formação de tumor e pode ser de origem benigna ou maligna (câncer) (Marinho, 2018).

O câncer é definido como uma doença crônica multicausal caracterizada pelo crescimento descontrolado de células e a proliferação de células anormais, que continuam a se multiplicar até que grandes quantidades de tecidos chamados de tumores sejam formados. Nos últimos anos, o câncer se tornou um problema de saúde pública global. Segundo dados da Organização Mundial da Saúde (OMS), o número estimado de cânceres no Brasil em 2016/2017 era de aproximadamente 600.000 casos (Souza; Gallon, 2017).

O termo câncer de cabeça e pescoço $(\mathrm{CCP})$ refere-se a um grupo de tumores heterogêneos que acometem o trato gastrointestinal superior, sendo o principal tipo histológico, o carcinoma espinocelular, responsável por cerca de $90 \%$ dos casos (Avelar et al., 2019).

É importante ressaltar que o câncer de cabeça e pescoço é mais expressivo em áreas com baixo desenvolvimento socioeconômico. Isso está relacionado aos hábitos de vida. Esses hábitos levam à exposição a longo prazo a fatores de risco e também ao diagnóstico tardio e dificuldade no acesso ao tratamento, sendo justificativa para o alto índice de letalidade (Pedrosa $e t$ al., 2019).

No método de tratamento, o foco está no uso de radioterapia e quimioterapia, dependendo da finalidade e do tipo de formação do tumor, a radioterapia e a quimioterapia podem ter efeitos auxiliares, neoadjuvantes, sistêmicos ou locais. Em relação às operações cirúrgicas, é fundamental remover os tumores com margens cirúrgicas livres, e cada tumor deve ser removido e deve

haver uma margem de tecido normal ao redor para evitar que células microscópicas causem recorrência. Isso é chamado de margem cirúrgica segura (Santos et al., 2019).

O tratamento mais comum para esse tipo de câncer é a cirurgia. Independentemente de a reconstrução ser realizada, a radioterapia e a quimioterapia podem ser aplicadas separadamente ou ao mesmo tempo. Para a escolha do tratamento, vários fatores são avaliados, como localização, tamanho e metástase do tumor, a fim de proteger os órgãos, função e questões estéticas (Avelar et al., 2019).

O trabalho tem por objetivo expor através da análise de artigos científicos as principais causas e tratamentos associados à Neoplasia de cabeça e pescoço.

\section{Metodologia}

Trata-se de uma revisão bibliográfica de caráter qualitativo que se baseia na produção científica a partir de estudos já publicados. A busca pelos textos foi realizada a partir das seguintes palavras-chaves indexadas no DECS (Descritores em Ciências da Saúde): "Câncer”; "Carcinoma de cabeça e pescoço"; "Tumores”; "Tratamento" nas plataformas Scientific Electronic Library Online e Biblioteca Virtual em Saúde (BVS).

Os critérios de inclusão foram pesquisas científicas publicadas de 2017 a 2021, publicados no idioma português, inglês e espanhol, que atendiam ao problema da pesquisa: Quais as principais causas e tratamentos associados à Neoplasia de cabeça e pescoço? Os critérios de exclusão foram trabalhos científicos com apenas resumos disponíveis, publicações duplicadas, artigos de relato de experiência, reflexivo, editoriais, comentários e cartas ao editor.

A partir do problema de pesquisa foram selecionados artigos publicados em periódicos nacionais e internacionais escolhidos a partir de levantamento realizado por meios dos descritores na biblioteca virtual Scientific Electronic Library Online, Biblioteca Virtual em Saúde e dissertações e teses.

Os artigos foram selecionados para leitura e foram incluídos na análise quantitativa artigos que se enquadravam no tema proposto do trabalho. 
Research, Society and Development, v. 10, n. 5, e55810515113, 2021

(CC BY 4.0) | ISSN 2525-3409 | DOI: http://dx.doi.org/10.33448/rsd-v10i5.15113

\section{Resultados e Discussão}

A Figura 1 ilustra os resultados deste trabalho que foram obtidos através da análise quantitativa de publicações sobre as principais causas e tratamentos para a Neoplasia de cabeça e pescoço na base de dados Scientific Electronic Library Online e Biblioteca Virtual em Saúde.

Figura 1: Fluxograma que apresenta o processo de seleção das publicações de Neoplasia de cabeça e pescoço, Teresina, Brasil, 2021.
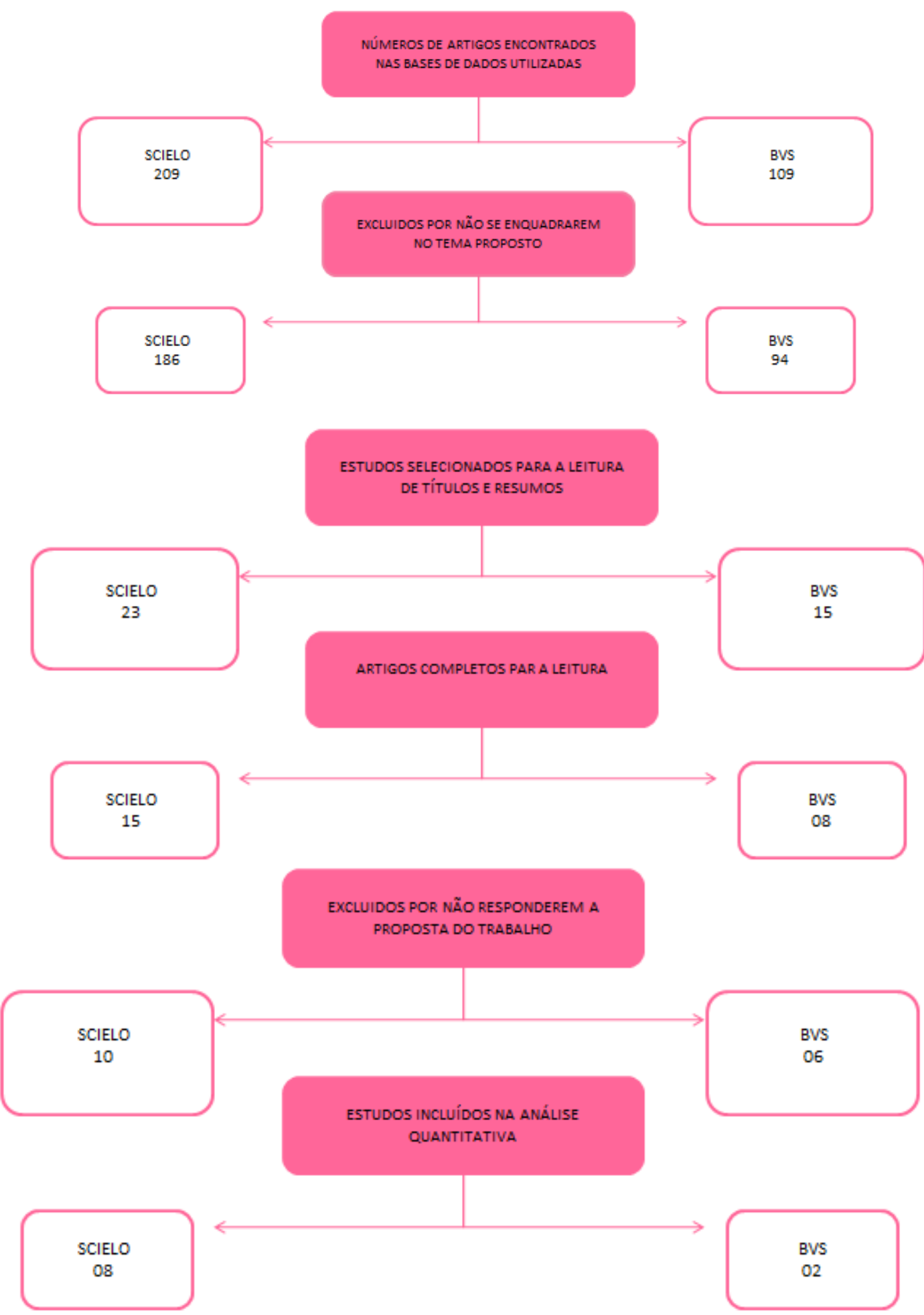

Fonte: Elaborado pelos autores. 
Os resultados deste trabalho foram obtidos através da análise quantitativa de publicações sobre Neoplasia de cabeça e pescoço encontrados nas bases de dados Scientific Electronic Library Online e Biblioteca Virtual em Saúde obtendo 209 artigos na Scielo e 109 artigos na BVS. Foram selecionados para a leitura de títulos e resumos o total de 23 e 15 artigos respectivamente e excluídos 186 artigos da Scielo e 94 da BVS por não se enquadrarem no tema proposto. Foi realizada a leitura completa de 15 artigos da Scielo e 08 artigos da BVS, onde 10 da Scielo e 06 da BVS não responderam a proposta do trabalho. Foram incluídos na análise quantitativa 08 artigos da Scielo e 02 artigos da BVS entre os anos de 2017 a 2021.

Quadro 1. Principais títulos e objetivos dos artigos utilizados na pesquisa, Teresina, Brasil, 2021.

\begin{tabular}{|c|c|c|}
\hline $\begin{array}{l}\text { AUTOR / ANO DE } \\
\text { PUBLICAÇÃO }\end{array}$ & TÍTULO & OBJETIVO \\
\hline $\begin{array}{l}\text { Avelar et al., } \\
2019 .\end{array}$ & $\begin{array}{c}\text { Fadiga em pacientes com câncer de cabeça e } \\
\text { pescoço submetidos à radioterapia: um estudo } \\
\text { prospectivo }\end{array}$ & $\begin{array}{c}\text { Identificar a frequência de fadiga e os } \\
\text { domínios afetados em pacientes com câncer } \\
\text { de cabeça e pescoço submetidos à } \\
\text { radioterapia, no início, meio e fim do } \\
\text { tratamento. }\end{array}$ \\
\hline $\begin{array}{l}\text { Da Silva et al., } \\
\quad 2020 .\end{array}$ & $\begin{array}{l}\text { Perfil Epidemiológico dos Pacientes com Câncer } \\
\text { de Cabeça e Pescoço em um Centro Oncológico } \\
\text { no Sul do Brasil. }\end{array}$ & $\begin{array}{l}\text { Determinar o perfil epidemiológico dos } \\
\text { pacientes com câncer de cabeça e pescoço } \\
\text { atendidos em um Centro Oncológico de } \\
\text { referência no Sul do Brasil no período de } \\
\text { janeiro de } 2013 \text { a dezembro de } 2018 \text {. }\end{array}$ \\
\hline $\begin{array}{l}\text { Leite et al., } \\
\quad 2021 .\end{array}$ & $\begin{array}{l}\text { A influência da associação de tabaco e álcool } \\
\text { no câncer bucal: revisão de literatura. }\end{array}$ & $\begin{array}{c}\text { Mostrar a influência da associação entre } \\
\text { tabagismo e etilismo no desenvolvimento do } \\
\text { câncer de boca. }\end{array}$ \\
\hline $\begin{array}{l}\text { Marinho, } \\
2018 .\end{array}$ & $\begin{array}{l}\text { Neoplasia de cabeça e pescoço: ocorrência de } \\
\text { tumores primários múltiplos }\end{array}$ & $\begin{array}{l}\text { Procurar-se-á entender o motivo de } \\
\text { aparecimento dos TPM, como acontecem, } \\
\text { quais são as populações de risco, as formas de } \\
\text { tratamento e o seu prognóstico. }\end{array}$ \\
\hline $\begin{array}{l}\text { Pedrosa et al., } \\
2019 .\end{array}$ & $\begin{array}{l}\text { Avaliação clínica dos sintomas de pacientes com } \\
\text { câncer de cabeça e pescoço. }\end{array}$ & $\begin{array}{l}\text { Avaliar os sintomas mais frequentes } \\
\text { apresentados pelos pacientes com câncer de } \\
\text { cabeça e pescoço e fatores associados. }\end{array}$ \\
\hline $\begin{array}{l}\text { Pereira } \text { et al., } \\
2020 .\end{array}$ & $\begin{array}{l}\text { Associação entre higiene oral e câncer de cabeça } \\
\text { e pescoço no Brasil. }\end{array}$ & $\begin{array}{l}\text { Determinar se a higiene bucal está associada } \\
\text { à ocorrência de câncer em cavidade oral e } \\
\text { cabeça e pescoço em uma amostra brasileira. }\end{array}$ \\
\hline $\begin{array}{l}\text { Santos et al., } \\
2021 .\end{array}$ & $\begin{array}{l}\text { Avaliação da sobrevida de pacientes com câncer } \\
\text { de cabeça e pescoço localmente avançado } \\
\text { tratados em um único centro. }\end{array}$ & $\begin{array}{l}\text { Mostrar a sobrevida global de uma grande } \\
\text { coorte de pacientes com câncer de cabeça e } \\
\text { pescoço localmente avançado tratados em } \\
\text { uma única instituição. }\end{array}$ \\
\hline $\begin{array}{l}\text { Santos et al., } \\
\quad 2019 .\end{array}$ & $\begin{array}{c}\text { Análise de Reações Adversas após o Tratamento } \\
\text { da Radioterapia em Adultos com Câncer de } \\
\text { Cabeça e Pescoço. }\end{array}$ & $\begin{array}{l}\text { Analisar as reações adversas após o } \\
\text { tratamento da radioterapia em adultos com } \\
\text { câncer de cabeça e pescoço. }\end{array}$ \\
\hline $\begin{array}{l}\text { Souza; Gallon, } \\
2017 .\end{array}$ & $\begin{array}{l}\text { Impacto do uso de dieta imunomoduladora e dieta } \\
\text { enteral em adultos, durante a quimioterapia e } \\
\text { radioterapia em pacientes com câncer de cabeça e } \\
\text { pescoço: uma revisão da literatura. }\end{array}$ & $\begin{array}{l}\text { Analisar, a partir de evidências científicas, se } \\
\text { o uso de imunonutrição é benéfico para } \\
\text { pacientes com câncer de cabeça e pescoço. }\end{array}$ \\
\hline $\begin{array}{l}\text { Véras et al., } \\
\quad 2019 .\end{array}$ & $\begin{array}{l}\text { Alterações orais e ingestão alimentar em } \\
\text { pacientes com câncer de cabeça e pescoço em } \\
\text { tratamento antineoplásico. }\end{array}$ & $\begin{array}{l}\text { O objetivo do presente estudo foi avaliar os } \\
\text { efeitos da terapia antineoplásica na } \\
\text { alimentação de pacientes com câncer de } \\
\text { cabeça e pescoço antes e após o tratamento, } \\
\text { utilizando parâmetros gustativos, salivares e } \\
\text { dietéticos. }\end{array}$ \\
\hline
\end{tabular}

Fonte: SCIELO e BVS. 
Desta forma, foi possível listar os principais resultados e conclusões dos estudos publicados na literatura, conforme o

Quadro 2.

Quadro 2. Resultados e conclusões dos artigos utilizados, Teresina, Brasil, 2021.

\begin{tabular}{|c|c|c|}
\hline AUTOR / ANO & RESULTADOS & CONCLUSÕES \\
\hline $\begin{array}{l}\text { Avelar et al., } \\
2019 .\end{array}$ & $\begin{array}{l}\text { Os participantes do estudo apresentaram como } \\
\text { características sociodemográficas prevalentes: sexo } \\
\text { masculino }(88,33 \%) \text {, faixa etária entre } 41-60 \text { anos } \\
(46,67 \%) \text {, baixa escolaridade / ensino fundamental } \\
\text { incompleto }(61,67 \%) \text {, aposentados }(41,67 \%) \text { e em } \\
\text { uso regular de álcool }(46,67 \%) \text { e fumo }(66,67 \%) \text {. } \\
\text { Esses achados corroboram os apresentados na } \\
\text { literatura nacional e internacional sobre pacientes } \\
\text { com CCP submetidos à radioterapia. Essa literatura } \\
\text { mostra que as características prevalentes são sexo } \\
\text { masculino, idade acima de } 40 \text { anos, baixa } \\
\text { escolaridade e uso regular de álcool e cigarro. }\end{array}$ & $\begin{array}{l}\text { Para um tratamento holístico é importante } \\
\text { avaliar os níveis de fadiga, assim como os } \\
\text { dos domínios afetados, ao longo do } \\
\text { tratamento, uma vez que, assim que } \\
\text { detectado o sintoma, os profissionais de } \\
\text { saúde podem começar a aplicar estratégias } \\
\text { de redução da fadiga , contribuindo assim } \\
\text { na condução do tratamento convencional, } \\
\text { mas também contribuindo para o bem- } \\
\text { estar do paciente. }\end{array}$ \\
\hline $\begin{array}{c}\text { Da Silva et al., } \\
2020 .\end{array}$ & $\begin{array}{l}\text { O gênero masculino foi o mais prevalente }(65,4 \%) \text {, } \\
\text { com média de idade acima dos } 50 \text { anos, baixa } \\
\text { escolaridade }(40 \%) \text {, baixa renda }(77,3 \%) \text {, sendo sua } \\
\text { grande maioria tabagistas }(72 \%) \text { e etilistas }(58,1 \%) \text {. } \\
\text { A região de cavidade oral }(26,3 \%) \text { foi o sítio mais } \\
\text { prevalente, a cirurgia mais realizada foi a } \\
\text { tireoidectomia total }(19,4 \%) \text { e o estadiamento mais } \\
\text { observado foi T2 }(30,8 \%) \text { N2 }(41,1 \%) \text {. Foram } \\
\text { encontradas complicações pós-radioterapia como a } \\
\text { radiodermite }(82,7 \%) \text { e, pósquimioterapia, náuseas } \\
\text { (81\%). }\end{array}$ & $\begin{array}{l}\text { Caracterizar o perfil dos pacientes com } \\
\text { câncer de cabeça e pescoço permite } \\
\text { conhecer os diversos acometimentos } \\
\text { advindos do tratamento e possibilita, } \\
\text { dessa maneira, maior qualidade e } \\
\text { direcionamento das ações de reabilitação. }\end{array}$ \\
\hline $\begin{array}{l}\text { Leite } \text { et al., } \\
\qquad 2021 .\end{array}$ & $\begin{array}{l}\text { Câncer, tumor ou neoplasia são palavras com } \\
\text { significado próximo para se referir a um conjunto } \\
\text { de cem doenças que acometem qualquer órgão, } \\
\text { cujo aspecto comum é a replicação rápida, } \\
\text { excessiva e anormal das células, induzindo a } \\
\text { formação de tumores nas células. }\end{array}$ & $\begin{array}{l}\text { A associação de uso do tabaco e ingestão } \\
\text { de álcool é a principal causa de câncer de } \\
\text { boca. Por meio do diagnóstico dessas } \\
\text { lesões, verificamos que os tipos de lesões } \\
\text { mais frequentes são leucoplasia e CCE } \\
\text { bucal, sendo a língua o local mais } \\
\text { atingido. }\end{array}$ \\
\hline $\begin{array}{l}\text { Marinho, } \\
2018 .\end{array}$ & $\begin{array}{l}\text { Nas últimas décadas apesar de haver progressos } \\
\text { científicos quer no diagnóstico como no tratamento } \\
\text { destas patologias, os TPM continuam a ser uma das } \\
\text { principais causas de morte nos pacientes com CCP. } \\
\text { A cancerização em campo é a teoria aceite pela } \\
\text { comunidade científica para explicar a ocorrência de } \\
\text { TPM. }\end{array}$ & $\begin{array}{l}\text { Com o aumento da incidência dos TPM, é } \\
\text { importante entender os mecanismos que } \\
\text { lhes antecedem (cancerização em campo), } \\
\text { identificar os hábitos de risco alertando } \\
\text { para os mesmos e proceder a vigilâncias } \\
\text { mais rigorosas aos pacientes que possuem } \\
\text { CPP. Desta forma é possível obter um } \\
\text { diagnóstico precoce, e melhorar o } \\
\text { prognóstico de TPM nestes pacientes. }\end{array}$ \\
\hline $\begin{array}{c}\text { Pedrosa et al., } \\
2019 .\end{array}$ & $\begin{array}{c}\text { A população do estudo é predominantemente } \\
\text { masculina }(67,5 \%) \text {, sendo pouco mais da metade } \\
\text { em uma faixa etária de } 60 \text { anos ou mais }(55 \%) \text {. A } \\
\text { maioria }(60 \%) \text { declarou-se da raça branca. Houve } \\
\text { predomínio do nível fundamental de escolaridade } \\
(60 \%) \text { e } 2,5 \% \text { dos entrevistados eram analfabetos. } \\
\text { Quanto ao estado civil, observou-se maior } \\
\text { prevalência de indivíduos casados, representando } \\
60 \% \text { da população. Em relação à localização do } \\
\text { tumor, houve predomínio de boca (30\%), seguido } \\
\text { de laringe (22,5\%). Dos entrevistados, 78,8 \% } \\
\text { estavam internados e 92,5\% não apresentaram } \\
\text { metástases. }\end{array}$ & $\begin{array}{l}\text { O presente estudo destinou-se a avaliar os } \\
\text { sintomas mais frequentes apresentados } \\
\text { pelos pacientes diagnosticados com } \\
\text { câncer de cabeça e pescoço utilizando a } \\
\text { escala msas. De fato, foi possível } \\
\text { identificar quais conjuntos de sintomas } \\
\text { foram mais frequentes e qual é o perfil } \\
\text { dos pacientes que apresentam esses } \\
\text { sintomas. }\end{array}$ \\
\hline
\end{tabular}




\begin{tabular}{|c|c|c|}
\hline $\begin{array}{c}\text { Pereira et al., } \\
2020 .\end{array}$ & 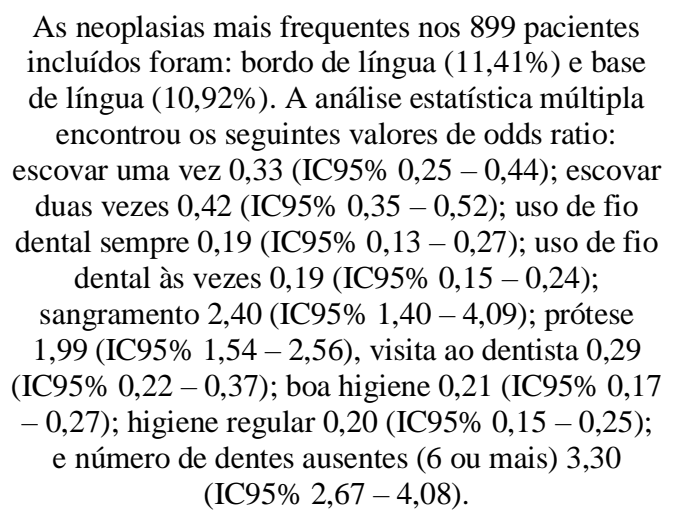 & $\begin{array}{l}\text { Esses dados mostraram que, na população } \\
\text { estudada, indicadores de boa higiene, } \\
\text { como escovar os dentes e uso do fio } \\
\text { dental, foram fatores de proteção para o } \\
\text { câncer de boca e cabeça e pescoço, } \\
\text { enquanto sangramento e muitos dentes } \\
\text { ausentes foram fatores de risco. }\end{array}$ \\
\hline $\begin{array}{c}\text { Santos et al., } \\
2021 \text {. }\end{array}$ & $\begin{array}{l}\text { Foram incluídos } 796 \text { pacientes com câncer de } \\
\text { cabeça e pescoço localmente avançado, sendo } 88 \% \\
\text { do sexo masculino, } 44 \% \text { com idade }>60 \text { anos e } \\
76 \% \text { no estágio IV. O tumor estava localizado na } \\
\text { cavidade oral (34\%), orofaringe ( } 27 \%) \text {, hipofaringe } \\
\text { (17\%) e laringe ( } 17 \%) \text {. Os grupos de tratamento } \\
\text { foram quimiorradioterapia }(39,7 \%) \text {, cirurgia } \\
\text { seguida de radioterapia ou quimiorradioterapia } \\
(26,3 \%) \text {, cirurgia seguida de radioterapia }(18,5 \%) \text { e } \\
\text { radioterapia isolada ( } 15,5 \%) \text {. Comparando as } \\
\text { variáveis clínicas entre os grupos de tratamento, } \\
\text { foram observadas diferenças significativas de idade } \\
\text { e estágio clínico. }\end{array}$ & $\begin{array}{l}\text { O presente estudo é a maior coorte de } \\
\text { câncer de cabeça e pescoço localmente } \\
\text { avançado de pacientes brasileiros para } \\
\text { avaliação dos desfechos do tratamento. } \\
\text { Embora houvesse diferenças clínicas } \\
\text { significativas entre os grupos cirúrgico e } \\
\text { radioterápico, a cirurgia ou a } \\
\text { quimiorradioterapia, como tratamento } \\
\text { inicial, não apresentaram diferenças } \\
\text { significantes em relação à sobrevida. }\end{array}$ \\
\hline $\begin{array}{c}\text { Santos et al., } \\
2019 .\end{array}$ & $\begin{array}{l}\text { As principais reações adversas encontradas foram } \\
\text { dor, mucosite, náusea, vômito, má ingestão, boca } \\
\text { seca, desidratação, alteração na voz e prurido. Não } \\
\text { houve diferenças significativas entre os grupos com } \\
\text { menos de dez e mais de dez sessões de radioterapia, } \\
\text { de acordo com as reações adversas. }\end{array}$ & $\begin{array}{l}\text { É necessário que os profissionais de saúde } \\
\text { tenham conhecimento dessas reações para } \\
\text { minimizar e tratar as complicaçôes, além } \\
\text { de acompanhamento contínuo com vistas } \\
\text { ao resgate das condições ideais de saúde, } \\
\text { contribuindo para a qualidade de vida e a } \\
\text { autoestima dos pacientes. }\end{array}$ \\
\hline $\begin{array}{c}\text { Souza; Gallon, } \\
2017 .\end{array}$ & $\begin{array}{l}\text { Observamos melhora no estado imunológico e } \\
\text { inflamatório, a prevenção de mucosite, } \\
\text { principalmente nos pacientes com tratamento de } \\
\text { quimioterapia e radioterapia com uso de } \\
\text { imunonutrição de arginina e ômega } 3 \text { no câncer de } \\
\text { cabeça e pescoço. }\end{array}$ & $\begin{array}{c}\text { Evidencia-se a importância do } \\
\text { acompanhamento nutricional, do início até } \\
\text { o final do tratamento, em pacientes com } \\
\text { câncer de cabeça e pescoço por } \\
\text { desenvolver perda de peso importante } \\
\text { com causas multifatoriais, aumentando o } \\
\text { risco nutricional, morbidade e } \\
\text { mortalidade. }\end{array}$ \\
\hline $\begin{array}{c}\text { Véras et al., } \\
2019 .\end{array}$ & $\begin{array}{l}\text { Ao comparar as etapas estudadas foi observado que } \\
\text { houve alterações no paladar e saliva }(\mathrm{p} \leq 0,99) \text { dos } \\
\text { pacientes, onde o fluxo salivar mostrou-se } \\
\text { correlacionado ao paladar da mesma forma que o } \\
\mathrm{pH} \text { também passou a influenciar significativamente } \\
\text { ( } \leq 0,99 \text { ) os limiares gustativos destes indivíduos. } \\
\text { Também foi possível observar a influência do fluxo } \\
\text { salivar entre os sabores doce ( } \leq \leq 0,4925) \text { e salgado } \\
(\mathrm{p} \leq 0,403) .\end{array}$ & $\begin{array}{l}\text { Os resultados sugerem que os efeitos } \\
\text { adversos decorrentes do tratamento } \\
\text { antineoplásico alteram a ingestão } \\
\text { alimentar do paciente e que independente } \\
\text { das alterações metabólicas da própria } \\
\text { doença, os efeitos adversos que ocorrem } \\
\text { na cavidade oral estão correlacionados } \\
\text { entre si e interferem diretamente na } \\
\text { alimentação, levando o paciente a reduzir } \\
\text { de forma significativa à quantidade dos } \\
\text { alimentos consumidos. }\end{array}$ \\
\hline
\end{tabular}

Fonte: SCIELO e BVS.

Baseando-se nos resultados e conclusões dos artigos utilizados, pode-se observar que o câncer é uma doença crônica com distribuição global, independentemente do nível de desenvolvimento local, representa um importante problema de saúde pública. É considerada a segunda causa de morte no mundo hoje. Os tumores de cabeça e pescoço podem ser relacionados a riscos associados, como o tabagismo, o etilismo e exposições ocupacionais. A maior parte é originada, com exceção dos tumores de pele, na mucosa das vias do trato gastrointestinal superior, principalmente boca, faringe e laringe. Nesta região anatômica 
destaca-se o câncer da cavidade oral e laringe como os mais incidentes no país (Pedrosa et al., 2019).

Os tumores benignos têm um bom prognóstico, enquanto os tumores malignos têm um prognóstico ruim e podem ser fatais. Além disso, esses dois tipos de tumores apresentam diferenças no crescimento e na aparência tanto macroscopicamente quanto microscopicamente. Em termos de crescimento, os tumores benignos crescem lenta e expansivamente (eles permanecem na posição inicial de proliferação celular), enquanto os tumores malignos crescem rápida e invasivamente (espalham-se para outras áreas e órgãos) (Marinho, 2018).

No câncer de cabeça e pescoço, fatores de risco genéticos e ambientais contribuem para sua etiologia, como beber e fumar, que são os fatores de risco mais relevantes para doenças do trato gastrointestinal superior. No que diz respeito ao cancro da tiroide, são múltiplas as razões consideradas fatores de risco, nomeadamente exposição à radiação, condições hormonais, obesidade, história familiar de cancro e ingestão de alimentos iodados. Na última década, devido à alta prevalência do papilomavírus humano (HPV), a incidência de câncer na cavidade oral aumentou, principalmente na parte inferior da língua e tonsilas, basicamente em menores de 45 anos. É considerado um fator de risco cada vez mais importante para esses tipos de câncer. O sexo oral frequente e um grande número de parceiros sexuais também comprovam esse aumento (Da Silva et al., 2020).

Segundo dados do Instituto Nacional do Câncer (INCA), o câncer de laringe ocorre principalmente em homens, pois está relacionado principalmente ao uso de álcool e tabaco e é um dos cânceres mais comuns que afetam a região da cabeça e pescoço. O número estimado de novos casos é de 7.350, dos quais 6.360 são do sexo masculino e 990 do feminino. Devido à sua localização, além dos efeitos colaterais que costumam ocorrer após as primeiras semanas de tratamento, esses tipos de câncer também podem impedir a mastigação, a secreção enzimas pelas glândulas salivares, bem como a ingestão de alimentos (Souza; Gallon, 2017).

O CCP afeta as áreas responsáveis pelas funções básicas, como respiração, deglutição e comunicação verbal. Essas complicações causadas pelo câncer e seu tratamento podem levar ao desmembramento e alterações físicas, como dificuldade de mastigação, disfagia, aspiração, alterações na fala e alterações estéticas, que afetam negativamente os aspectos físicos e sóciopsicológicos desses pacientes (Avelar et al., 2019).

O câncer de cabeça e pescoço ocupa o sexto lugar entre os tumores mais comuns, e estima-se que 780.000 novos casos ocorram em todo o mundo a cada ano. As partes anatômicas contidas neste grupo de tumores constituem a cavidade oral, incluindo mucosa oral, gengiva, palato duro, língua e base da língua; faringe, incluindo: orofaringe, nasofaringe, hipofaringe; cavidade nasal e seios paranasais, laringe e glândulas. Aproximadamente $40 \%$ ocorrem na cavidade oral, $15 \%$ ocorrem na faringe, $25 \%$ ocorrem na laringe e o restante ocorre em outras partes (Souza; Gallon, 2017).

O Quadro 3 demonstra as principais estruturas anatômicas atingidas pelas neoplasias malignas de cabeça e pescoço. 
Quadro 3: Apresenta as principais estruturas anatômicas em que são localizadas as neoplasias da região da cabeça e pescoço.

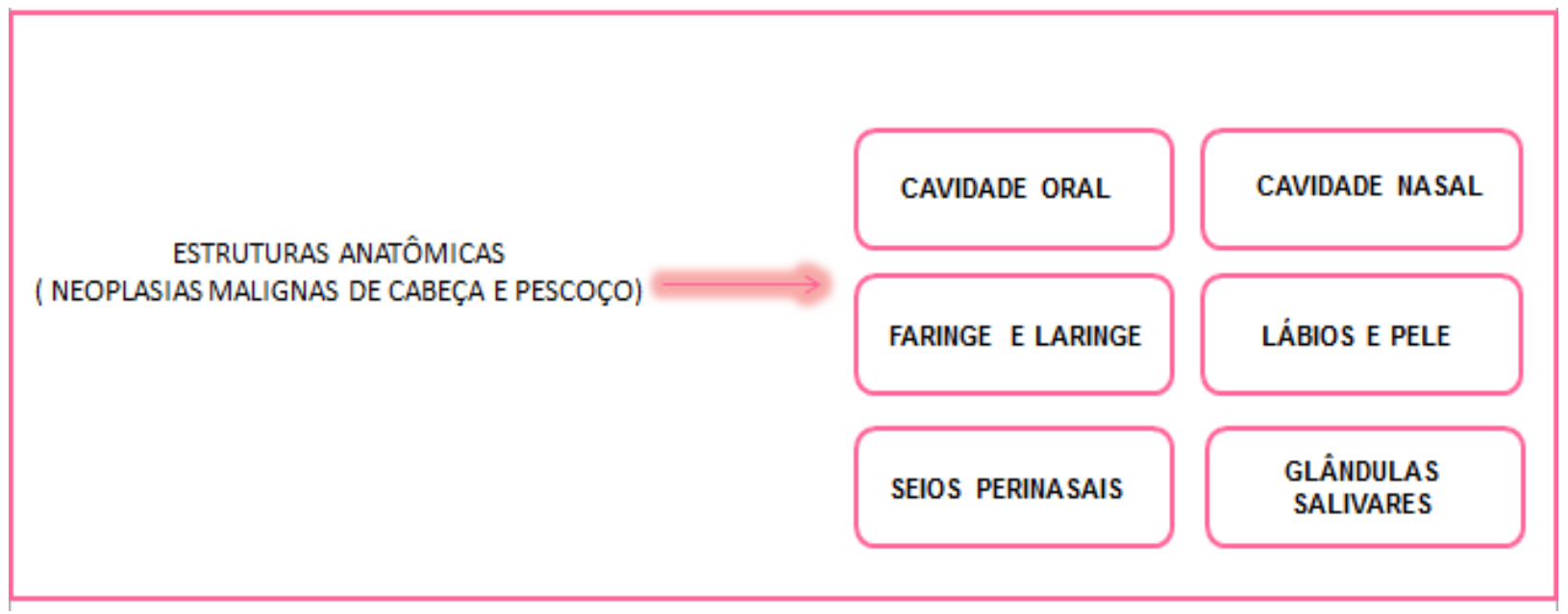

Fonte: Adaptado de Marinho (2018).

$\mathrm{O}$ câncer de cabeça e pescoço (CCP) está listado como o sexto câncer mais comum no mundo, abrangendo vários tumores malignos localizados em diferentes estruturas anatômicas de cabeça e pescoço, entre elas: Cavidade oral, (incluindo língua, assoalho da boca, vestíbulo, processo alveolar, palato duro, palato mole, úvula, triângulo molar posterior e área jugal), cavidade nasal, faringe e laringe, lábios, seios da face, glândulas salivares e pele. A incidência anual desse câncer ultrapassa 550.000 novos casos e a taxa de mortalidade anual ultrapassa 300.000 casos. Quanto à prevalência em termos de gênero, a correlação com os homens é maior, com proporção de 2: 1 a 4: 1 (Marinho, 2018).

Nos Estados Unidos, o carcinoma de cabeça e pescoço representa cerca de 3\% de todos as neoplasias. Baseado nos dados do Instituto Nacional do Câncer (INCA) brasileiro, em 2017 houve aproximadamente 23.000 novos casos de neoplasia de cabeça e pescoço. Embora tenha tido melhoria nos desfechos com a terapia multimodal para a doença localmente avançada, ainda existe uma alta taxa de mortalidade para essa patologia (Santos et al., 2021).

Evidências epidemiológicas mostram que a incidência de CCP aumenta com a idade. Na Europa, 98\% dos pacientes têm mais de 40 anos. Este tipo de tumor é raro na população jovem. Cerca de $6 \%$ da doença ocorre em indivíduos com menos de 40 anos, mas em alguns países a incidência vem aumentando e os mecanismos envolvidos na carcinogênese desses pacientes não são bem esclarecidos. Quanto à localização, cerca de $40 \%$ do CCP acomete a cavidade oral, $15 \%$ da faringe, $25 \%$ da laringe e o restante se localizam nas demais partes (glândulas salivares e tireoide). Vários outros autores relataram que a língua, o assoalho da boca e o lábio inferior são as áreas mais comuns de câncer oral (CO). Em relação ao tipo histológico, o mais comum é o carcinoma espinocelular, prevalente em mais de 90\% dos casos de câncer (Santos et al., 2019).

Acredita-se que os tumores de cabeça e pescoço causam importantes alterações físicas, motoras e sensorial nos pacientes, o que reduz muito sua qualidade de vida e afeta negativamente sua saúde mental. Portanto, independentemente da fase e do tipo de tratamento, é importante avaliar a ocorrência, intensidade e frequência dos sintomas (Pedrosa et al., 2019).

$\mathrm{O}$ tratamento desses pacientes pode comprometer a qualidade de vida, causando alterações como mucosite (inflamação da mucosa oral), boca seca, fadiga e dermatite por radiação (alterações na pele), que podem causar danos à mastigação, deglutição e até mesmo danos na fala. Na maioria dos casos, essas alterações podem levar à perda de peso e desnutrição devido à morbidade decorrente do tratamento, levando a graves declínios funcionais. Dentre as alterações e declínios funcionais causados pelo tratamento do câncer de cabeça e pescoço, destacam-se o linfedema, a síndrome do ombro caído, a paralisia facial, o trísmo (diminuição da abertura da boca) e a paralisia das cordas vocais (Da Silva et al., 2020).

O tratamento mais importante no CCP é o uso de radioterapia (RT), que utiliza energia eletromagnética ou de ionização 
de partículas, que pode causar efeitos químicos e biológicos para impedir a replicação de células neoplásicas. Por outro lado, é considerado o tratamento de escolha para os CCPs e tem sido utilizado para tratar lesões malignas de cabeça e pescoço, inibir metástases e melhorar significativamente a sobrevida dos pacientes. Pode ser usado sozinho ou em combinação com cirurgia e / ou quimioterapia (Santos et al., 2019).

Se o diagnóstico for positivo para câncer, é necessário verificar o estágio da neoplasia para iniciar o tratamento. Também é imprescindível avaliar se as células cancerígenas atingiram outros órgãos (denomina-se metástase quando o carcinoma atinge além de onde começou) (Leite et al., 2021).

A cirurgia e a radioterapia mostraram-se os tratamentos mais eficazes para esses tipos de câncer, seguidos da quimioterapia. No entanto, independentemente do método escolhido, pode haver sequelas ou efeitos adversos. A característica da radioterapia é o uso de raios ionizantes para afetar o DNA das células cancerosas, fazendo com que as células cancerosas percam repetidamente sua capacidade reprodutiva e morram. Essa terapia é extremamente importante no tratamento de tumores de cabeça e pescoço, mas, apesar de seus benefícios, também pode destruir células saudáveis, resultando em efeitos colaterais. Essas complicações ocorrem em quase $90 \%$ dos pacientes acometidos pelo câncer de cabeça e pescoço, e isso acontece, além das circunstâncias específicas de cada paciente, mas depende também da dose diária de radioterapia e da área de irradiação. Como dose terapêutica, costuma ser utilizada entre 50 Gy e 70 Gy por 5 a 7 semanas, dividida em 2 Gy por dia, 5 dias por semana, mas a dose e a frequência dependem da situação clínica específica e do estágio do tumor (Véras et al., 2019).

Os tratamentos para esses tipos de câncer podem incluir ressecção cirúrgica, quimioterapia, radioterapia, terapia hormonal ou uma combinação de múltiplas modalidades de tratamentos, resultando em alterações na função e na qualidade de vida. Portanto, a atenção ao paciente com câncer de cabeça e pescoço deve ser multidisciplinar, composta por equipe de médicos, dentistas, fisioterapeutas, enfermeiros, fonoaudiólogos, entre outros profissionais (Da Silva et al., 2020).

O principal fator etiológico das neoplasias de cabeça e pescoço é o fumo, e a associação entre os dois pode ser aumentada pelo consumo de bebidas alcoólicas. Entretanto, como o câncer é uma doença multifatorial, existem outros fatores associados com o seu desenvolvimento, como fatores nutricionais, mutações herdadas e condições imunológicas. Alguns vírus têm potencial carcinogênico, incluindo o papilomavírus humano (HPV). O efeito do HPV já foi associado com o câncer cervical e tem associações com carcinomas de cabeça e pescoço, especialmente na orofaringe. Fatores sociais e estilo de vida, além de serem fatores de risco, alteram o prognóstico da doença. Mesmo com tantos fatores já conhecidos e estudados, novas relações e hipóteses precisam ser investigadas para controlar os outros possíveis fatores etiológicos (Pereira et al., 2020).

\section{Conclusão}

O CCP é considerado uma das neoplasias mais comuns mundialmente. Pode afetar diversas partes anatômicas do sistema gastrointestinal superior. É um câncer mais comumente encontrado em homens acima dos 40 anos. É uma doença que impacta diretamente na qualidade de vida dos pacientes, podendo impedi-los de ingerir alimentos e impossibilita-los de falar. Os tratamentos mais utilizados envolvem procedimentos cirúrgicos, quimioterapia e radioterapia. Esses pacientes devem ser acompanhados por diversos profissionais da saúde, pois essa patologia altera diversas funções do paciente.

Infelizmente, a neoplasia de cabeça e pescoço afeta milhares de pessoas e possui um impacto significativo na qualidade de vida desses pacientes. São necessários estudos científicos mais aprofundados sobre esse câncer e o desenvolvimento de medidas de prevenção para a população em geral, conscientizando-os sobre como o tabagismo, o consumo de bebidas alcoólicas e a infecção pelo o HPV podem ser causas para o desenvolvimento dessa neoplasia maligna, bem como o desenvolvimento de novas terapias mais eficazes e que minimizem os sintomas dessa doença. 
Research, Society and Development, v. 10, n. 5, e55810515113, 2021

(CC BY 4.0) | ISSN 2525-3409 |DOI: http://dx.doi.org/10.33448/rsd-v10i5.15113

\section{Referências}

Avelar, J., Nicolussi, A. C., Toneti, B. F., Sonobe, H. M., \& Sawada, N. O. (2019). Fadiga em pacientes com câncer de cabeça e pescoço em tratamento radioterápico: estudo prospectivo. Revista latino-americana de enfermagem, 27, e-3168.

Da Silva, F. A., Roussenq, S. C., De Souza Tavares, M. G., De Souza, C. P. F., Mozzini, C. B., Benetti, M., \& Dias, M. (2020). Perfil epidemiológico dos pacientes com câncer de cabeça e pescoço em um centro oncológico no sul do Brasil. Revista Brasileira de Cancerologia,66(1), e-08455.

Leite, R. B., Marinho, A. C. O., Costa, B. L., Laranjeira, M. B. V., Araújo, K. D., \& Cavalcanti, A. F. (2021). A influência da associação de tabaco e álcool no câncer bucal: revisão de literatura. J Bras Patol Med Lab, 57: 1-5.

Marinho, P. A. V. (2018). Neoplasia de cabeça e pescoço: ocorrência de tumores primários múltiplos (Doctoral dissertation).

Pedrosa, T. M., de Freitas Martins, T. C., de Souza, A. L. L. P., da Silva, D. G. F., Moura, S. F., Muzi, C. D., \& Guimarães, R. M. (2019). Avaliação clínica dos sintomas de pacientes com câncer de cabeça e pescoço. Avances en Enfermería, 37(2), 158-168.

Pereira, N. F., López, R. M., Toporcov, T. N., Schmerling, C. K., Cicco, R. D., Michel-Crosato, E., \& Biazevic, M. G. H. (2020). Association between oral hygiene and head and neck cancer in Brazil. Revista Brasileira de Epidemiologia, 23, e-200094.

Santos, F. M. D., Viani, G. A., \& Pavoni, J. F. (2021). Avaliação da sobrevida de pacientes com câncer de cabeça e pescoço localmente avançado tratados em um único centro. Brazilian Journal of Otorhinolaryngology, 87(1), 3-10.

Santos, J. N. A., Matos, F.R., Santana, I. T. S., \& Matos, A. L. P. (2019). Análise das reações adversas após o tratamento com radioterapia em adultos com câncer de cabeça e pescoço. Revista Brasileira de Cancerologia, 65(4), e-12648.

Souza, J. A. D., \& Gallon, C. W. (2017). Impacto do uso de dieta imunomoduladora e dieta enteral em adultos, durante a quimioterapia e radioterapia em pacientes com câncer de cabeça e pescoço: uma revisão da literatura. Braspen J, 273-281.

Véras, I. D., dos Santos, A. F., Ferreira, S. M. S., de Oliveira, C. R. R., \& da Costa, J. G. (2019). Alterações orais e ingestão alimentar em pacientes com câncer de cabeça e pescoço em tratamento antineoplásico. Diversitas Journal, 4(2), 566-579. 